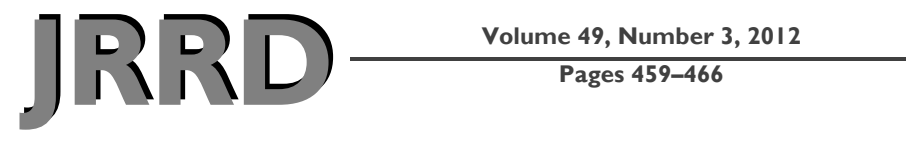

\title{
Is it important to position foot in subtalar joint neutral position during non-weight-bearing molding for foot orthoses?
}

\author{
Winson C. C. Lee, PhD; ${ }^{1}$ Christina K. L. Lee, MPhil; ${ }^{1}$ Aaron K. L. Leung, PhD; ${ }^{1 *}$ Stephen W. Hutchins, PhD $^{\mathbf{2}}$ \\ ${ }^{1}$ Department of Health Technology and Informatics, The Hong Kong Polytechnic University, Hung Hom, Kowloon, \\ Hong Kong; ${ }^{2}$ Centre for Health, Sport and Rehabilitation Sciences Research, University of Salford, Salford, United \\ Kingdom
}

\begin{abstract}
When taking molds for foot orthoses, it is accepted practice to position the subtalar joint in its neutral position. However, foot orthoses have no contact with the talus, and this leads to a hypothesis that as long as there is correction available to appropriately align the forefoot relative to the hindfoot when taking a mold, changes in subtalar joint angles do not lead to significant alterations in the plantar surface shapes of the molds taken. This study tested this presumption with 20 subjects between 22 and 46 years old. During non-weightbearing casting, the subtalar joints were aligned at positions of $4^{\circ}$ of eversion, $2^{\circ}$ of eversion, $2^{\circ}$ of inversion, and in neutral. At each orientation, forces were applied over the forefoot such that the metatarsal heads were aligned with the rearfoot. Digital scanning was used to analyze the shape of each negative mold. There were significant changes in projection volume in different subtalar joint orientations. However, the changes in arch heights, navicular height, and protrusion were insignificant and very small. It is therefore suggested that as long as the forefoot and hindfoot are appropriately aligned, variations in the orientation of the subtalar joint would be acceptable.
\end{abstract}

Key words: alignment, CAD/CAM, casting, digital scanning, foot impression, foot orthosis, foot shape, insole, non-weightbearing, subtalar joint.

\section{INTRODUCTION}

Foot orthoses are commonly used to absorb shock and support the medial longitudinal arch in an attempt to relieve foot pain. A foot orthosis is conventionally made by first obtaining a foot mold, which can be obtained in either a non-weight-bearing position, using materials such as plaster of Paris bandages or low-temperature formable thermoplastics, or a semi-weight-bearing position [1-2], which can also use disposable foam boxes or scanning devices. A positive model is then created and shape-modified, and finally a foot orthosis is manufactured using appropriate materials. Although computeraided design/computer-aided manufacturing has been used since the 1990s, these conventional foot molding techniques are still commonly used because orthotists are able to rely on their clinical experience to align different joints of the foot. In addition to the choice of materials, the foot alignment position during the molding procedure has been shown to be one of the most important factors for the quality of foot orthoses [1-3].

The undertaking of foot molds has been regarded as an art rather than a true scientific discipline [4]. Molds taken in a non-weight-bearing position allow the orthotist to alter the alignment of different joints of the foot during the molding procedure in order to correct the forefoot and capture the rearfoot-forefoot relationship at a

\footnotetext{
Abbreviation: ICC = intraclass correlation coefficient.

*Address all correspondence to Aaron K. L. Leung, PhD; Department of Health Technology and Informatics, The Hong Kong Polytechnic University, Hung Hom, Kowloon, Hong Kong, China; 852-2766-7676; fax: 852-2362-4365.

Email: aaron.leung@polyu.edu.hk

http://dx.doi.org/10.1682/JRRD.2011.03.0049
} 
chosen rearfoot position. This is more difficult to achieve using semi-weight-bearing methods. The non-weightbearing approach is recommended when the forefoot-torearfoot relationship is important, such as in a functional orthosis [2]. If the midtarsal joint is flexible, it is generally agreed that gentle forces should be applied to orientate the forefoot so that the plane of the metatarsal heads are perpendicular to the vertical bisector of the calcaneus [5]. This alignment, reported with high reliability [6], follows the normal forefoot-to-hindfoot alignment at the point of midstance [7], gives stability of the foot orthoses within the shoes, and locks the midtarsal joint to prepare the foot in push-off phase. The current guidelines available for taking foot molds recommend that the subtalar joint be orientated at its neutral position [7-8]. This approach has been questioned because more recent biomechanical studies have indicated that the subtalar joint is everted during relaxed standing [9-11] and the early stance phase of the gait [12-16]. In spite of this controversy, it is the common practice of orthotists to carefully align the subtalar joint in a neutral position during foot molding. A common belief is that the foot will rest on the orthosis at a subtalar joint neutral position if the joint is aligned in such a way during the foot mold process.

In aligning the subtalar joint in its neutral position when taking a foot mold, two lines are normally drawn: one bisecting the calcaneus and the other bisecting the posterior aspect of the lower third of the tibia. Forces should then to be applied to the foot such that the two lines are co-linear. However, the reason for paying attention in aligning the subtalar joint at this specific angle is not well understood. While the subtalar joint refers to the articulation between the calcaneus and talus, foot orthoses have little or no contact with the talus. This leads to a hypothesis that as long as the forefoot is appropriately aligned with respect to the hindfoot, changes in subtalar joint angle during non-weight-bearing foot molding procedures do not lead to significant alterations in the shapes of the positive model and therefore also the foot orthosis superstructure tomography. However, note that subtalar joint motions might result in movements of other foot bones caused by ligament tensions leading to changes in foot shapes [17]. It requires certain skills and experience for clinicians to maintain the subtalar joint in neutral while both taking molds and also simultaneously correcting the forefoot. Finding out whether aligning the subtalar joint in neutral is necessary during non-weightbearing molding would be important.
Evidence in the literature demonstrates that differences can occur in the shape of foot molds obtained when comparing different molding techniques such as those when the patient is non-weight-bearing or semi-weightbearing [18] or with differences in forefoot-rearfoot alignment [19]. However, due to lack of evidence in the literature, little understanding exists regarding how the subtalar joint should be aligned during non-weight-bearing molding and its rationale.

In this study, we obtained non-weight-bearing foot molds with the subtalar joint aligned at four different orientations. Different orientations of the subtalar joints required different levels of forces to be applied to align the forefoot with respect to the hindfoot. To accurately evaluate the foot shape, we scanned the foot models and used a computer program to quantify various foot parameters. The aim of this study was to reveal whether there were changes in foot shape obtained in molds taken when the subtalar joint was at different orientations. We also assessed the reliability of the use of goniometers to measure subtalar joint angles. We hypothesized that different subtalar joint orientations would not change the foot shape as long as the forefoot and hindfoot were appropriately aligned. This would provide useful information regarding non-weight-bearing foot molding techniques for the field of foot orthotics.

\section{METHODS}

\section{Subjects}

Twenty voluntary adult subjects (8 male and 12 female) between 22 and 46 years old (mean: 28.3 years) participated in this study. The subjects had not sustained any lower-limb injuries or incurred any rigid foot deformities during the previous 12 months.

\section{Subtalar Joint and Forefoot-Hindfoot Orientations, Foot Molding, and Digital Scanning}

We took a series of non-weight-bearing foot molds when the subjects were lying prone on an examination table. The orthotist first aligned the subtalar joint of the right foot of each subject at a neutral position to obtain a foot mold. Another three molds were then taken on the same foot with the subtalar joints aligned at $4^{\circ}$ of eversion, $2^{\circ}$ of eversion, and $2^{\circ}$ of inversion. At each subtalar joint orientation, the subject placed the forefoot such that a line joining the first and fifth metatarsal heads was 
perpendicular to the vertical bisector of the calcaneus as measured by a goniometer. The orthotist adjusted the subtalar joint angles and forefoot-hindfoot spatial relationships by applying forces over the sulcus between the toes and the metatarsal heads and applied forces over the sulcus to prevent distortion of the shape of impression at the midfoot and hindfoot areas.

We drew two lines: one bisecting the calcaneus and the other bisecting the posterior aspect of the lower third of the tibia on the right leg. We then positioned the subtalar joints at different pre-determined angles based on the angles measured between the two lines by a goniometer [20]. Foot molds were obtained using Orfit low-temperature thermoplastic material (Orfit Industries; Wijnegem, Belgium) [6], which the orthotist softened after heating and wrapped around the foot. Using low temperature thermoplastic has been shown to be appropriate for use during foot impression taking for manufacturing foot orthoses in a previous study [6]. The semitransparent thermoplastic material allowed the calcaneal bisection line to be visible. We filled the resulting thermoplastic positive foot molds with plaster of Paris and scanned the positive plaster models with a 3dimensional foot scanner (INFOOT, I-Ware Laboratory; Japan). The scanner consists of eight charge coupled device cameras and four laser projectors and is capable of scanning a foot in $10 \mathrm{~s}$ with a resolution of $1.0 \mathrm{~mm}$. We placed the plaster of Paris models on the scanner with the calcaneal line vertical to the scanner surface and with the forefoot at right angles to this line and scanned the plantar surface profile in this static position.

To assess the reliability of the measurements of subtalar joint angles using goniometers, we marked the two bisection lines twice. We also measured the subtalar joint angles twice during relaxed standing when a consistent amount of ground reaction force was acting on the foot. During measuring, each subject stood upright with their feet positioned with toe-in and toe-out angles; we also measured the distance between the heels following the standardized approach of McPoil et al. [5].

\section{Quantification of Foot Shape}

We developed a computer program to identify various foot parameters (Figure 1). We identified the anteroposterior $(y)$ axis by a line joining the most medial points of the anterior and posterior one-third of the plaster of Paris model. The medio-lateral $(x)$ axis was perpendicular to the $y$-axis and tangent to the most posterior point of the model. The vertical axis ( $\mathrm{z}$ ) was perpendicular to the supporting surface of the plaster of Paris model, with $z=$ 0 defined as a plane at the supporting surface. We divided the foot models into six regions, namely medial and lateral forefoot, midfoot, and heel regions, as defined in previous studies (Figure 1). Similar approaches to dividing the foot scans into forefoot, midfoot, and heel regions have been used previously [18].

We studied the following parameters:

1. Projection volume under six foot regions $\left(\mathrm{mm}^{3}\right)$. This was the volume between supporting floor surface and the plantar foot surface over one of the six divided regions. These provided information on the 3dimensional spatial orientation of the different regions of the foot relative to the floor and revealed whether different subtalar joint orientations would lead to the collapse or elevation of a particular foot region.

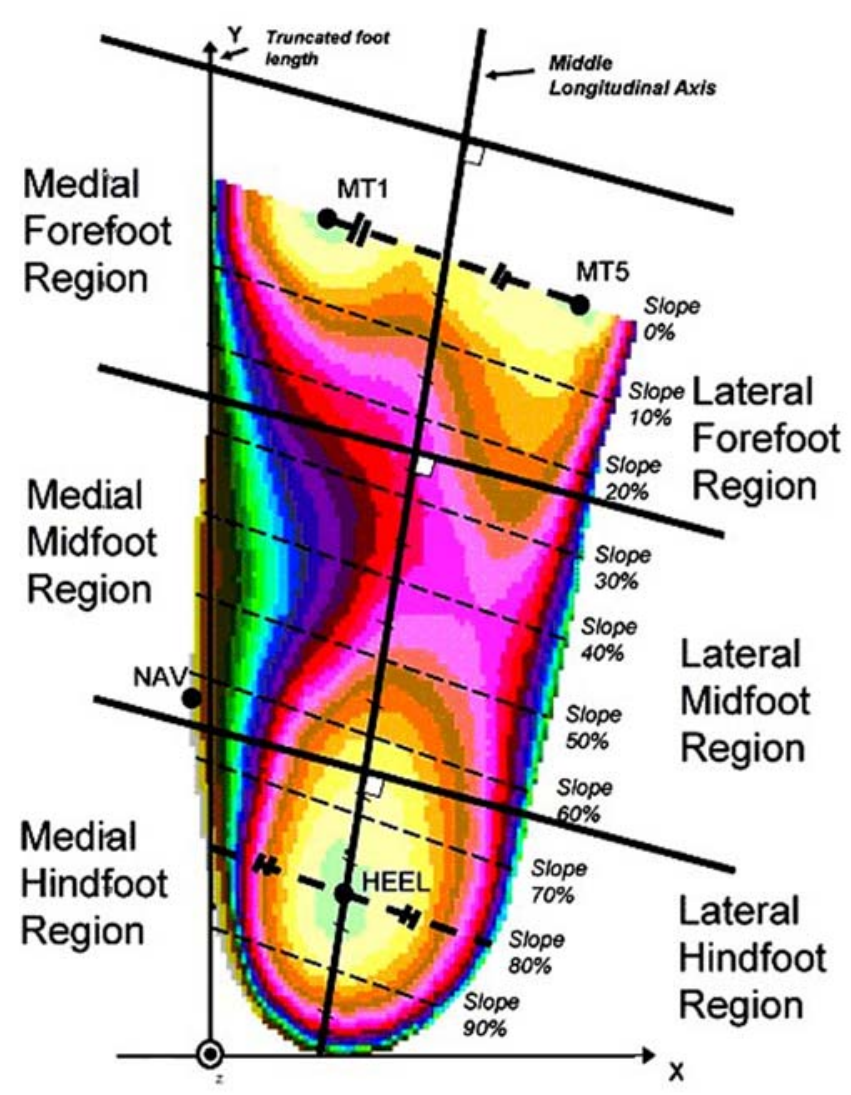

Figure 1.

Typical foot model with definition of some foot parameters. HEEL = heel contact point, MT1 = first metatarsal contact point, MT5 = fifth metatarsal contact point, NAV = navicular. 
2. Medio-lateral slopes $\left({ }^{\circ}\right)$. The model (excluding the toes) was evenly divided into 10 slices along the long axis of the foot (Figure 1). The medio-lateral slope of each slice was the slope of the regression line of all the data points at the central region at the corresponding slice. We obtained the central region based on the ratio of the distance between the first and fifth metatarsal contact points and the medio-lateral dimension at this cross-section. Similar approaches in investigating the medio-lateral slopes of the foot have been used previously [17]. These parameters showed the angulations of the plantar foot surface with respect to the floor at various longitudinal locations.

3. Navicular protrusion and height $(\mathrm{mm})$. We took navicular protrusion to be the $x$-coordinate of the most medial point at the middle one-third of the foot image. We regarded the corresponding $z$-coordinate of that point as the navicular height. These parameters therefore related to the position and the level of the bony prominence of the navicular.

4. Medial and lateral longitudinal arch height (mm). We took the arch heights to be the $z$-coordinate of the highest point at the medial and lateral mid-foot region. These parameters therefore related to the heights of arch support features demonstrated by the model.

\section{Data Analysis}

We used the intraclass correlation coefficient (ICC) $(3,1)$ to determine the intrarater reliability of the measurement of subtalar joint angles. We used repeated-measures analysis of variance (95\% confidence interval) to determine whether significant differences existed among different subtalar joint orientations. We subsequently performed post-hoc Bonferoni tests to compare each testing condition. We regarded a $p$-value smaller than 0.05 as statistically significant. When performing multiple comparisons among the four testing conditions, we performed Bonferoni correction adjusting $\alpha$ to be 0.008 .

\section{RESULTS}

The ICC $(3,1)$ for the repeated markings and measurements of subtalar joint angles was 0.91, indicating high repeatability. Figures $\mathbf{2}$ to $\mathbf{4}$ show the projection volumes, medial-lateral slopes, arch heights, and navicular positions.

The projection volume under the medial hindfoot regions in the $2^{\circ}$ of eversion position was significantly

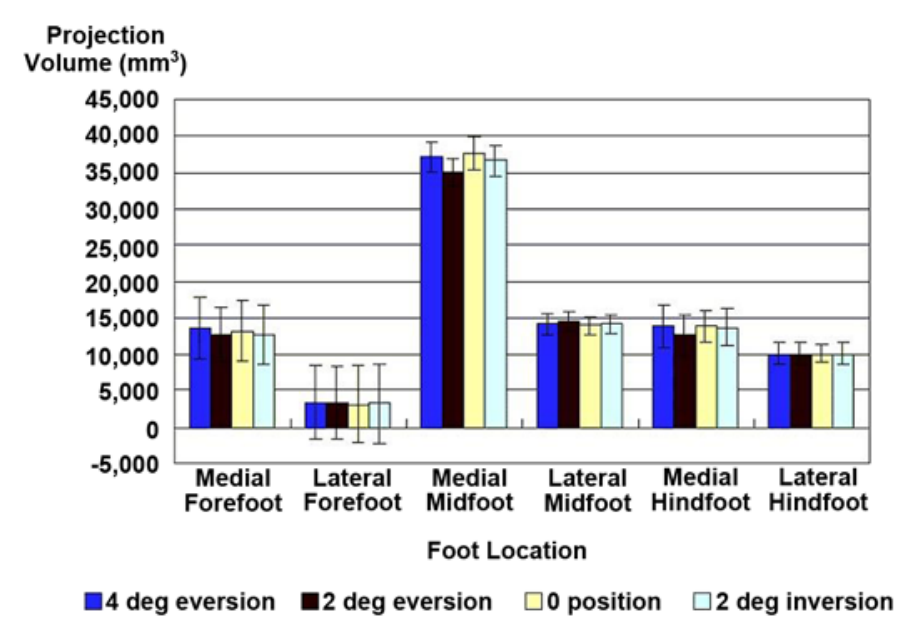

Figure 2.

Projection volume at different foot regions at different subtalar joint orientations.

lower than the neutral position $(-9.0 \%)$ and with $2^{\circ}$ of inversion (-7.4\%) (Figure 2). The $2^{\circ}$ of eversion position also produced significantly lower projection volume under the medial mid-foot than the neutral position $(-7.5 \%)$ and $2^{\circ}$ of inversion (-4.9\%). This corresponded well with the smallest medio-lateral slope found at 30 and 40 percent of the foot length with $2^{\circ}$ of subtalar joint eversion (Figure 3 ). When the subtalar joint was further everted to $4^{\circ}$, however, the projection volume under the medial forefoot region $(+8.0 \%)$ and the medial midfoot region $(+9.0 \%)$ were significantly larger than the condition of the $2^{\circ}$ of eversion.

We found no significant differences in other parameters. As far as arch height, navicular height, and protrusion are concerned (Figure 4), the differences were small and insignificant among the four different joint angles. The average changes in navicular protrusion, metatarsal width, and medial and lateral arch height were less than $0.6 \mathrm{~mm}$. The average changes in navicular height were less than $1.5 \mathrm{~mm}$.

\section{DISCUSSION}

The tested range of the subtalar joint orientations $\left(2^{\circ}\right.$ of inversion to $4^{\circ}$ of eversion) was smaller than its passive full range of motion. This is because large subtalar joint angles are unlikely to be produced during semiweight-bearing and non-weight-bearing foot molding 


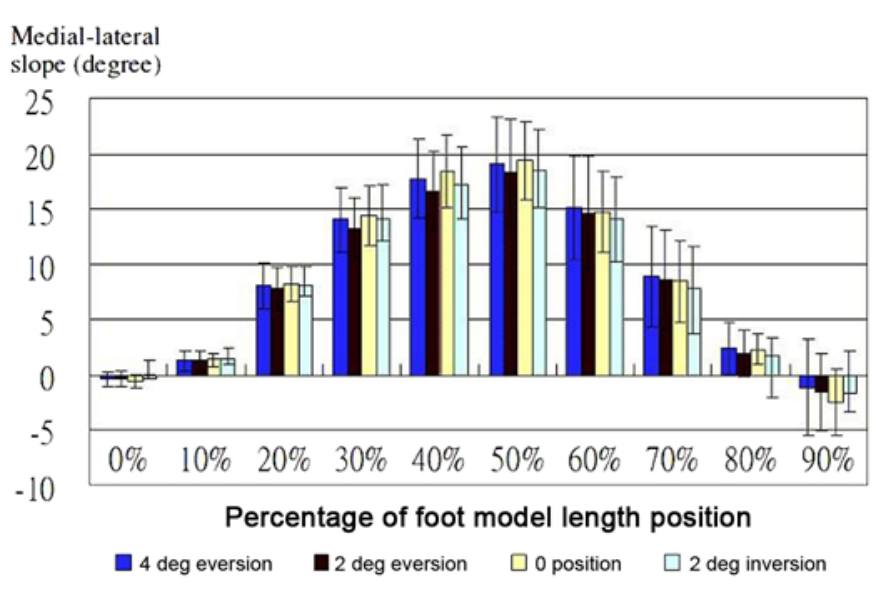

Figure 3.

Medio-lateral slopes at different subtalar joint orientations.
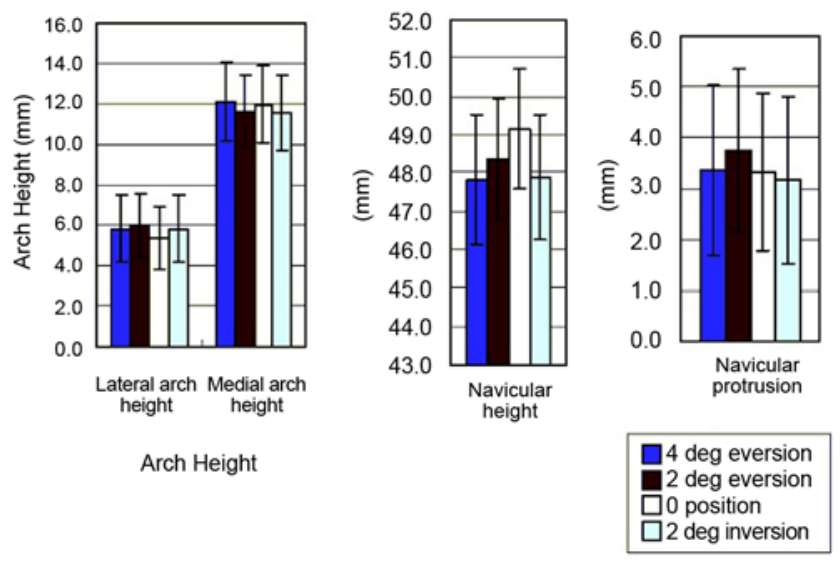

Figure 4.

Dimensional parameters at different subtalar joint orientations.

techniques. During relaxed standing, this study revealed a small (average of $0.7^{\circ}$ ) eversion angle, which was consistent with previous studies [10]. During stance phase of the gait, a previous study reported an average of a small $3.5^{\circ}$ of peak eversion and a $3.1^{\circ}$ of peak inversion [21].

Subtalar joint eversion is usually accompanied with abduction and dorsiflexion of the calcaneus in open kinetic chain motions. These motions at the subtalar joint are collectively called pronation of the subtalar joint and could affect motions at more proximal and distal joints. Subtalar joint pronation during weight bearing would induce internal rotation of the tibia [17]. Foot pronation also allows the foot to become more flexible, making it able to adapt to uneven terrain [5]. However, little is known about how subtalar joint pronation exactly affects foot shape obtained by non-weight-bearing molds.

When the subtalar joint was positioned at $2^{\circ}$ of eversion, we found that the projection volumes under the medial midfoot and hindfoot regions of the plaster of Paris models were significantly lower than the other three subtalar joints positions. The abduction and dorsiflexion of the calcaneus accompanied with eversion would explain the lowering of medial longitudinal arches. However, when the subtalar joint was further everted from $2^{\circ}$ to $4^{\circ}$, on the contrary, we found significant increases in projection volumes under the medial midfoot and forefoot regions. This can be explained by the action of aligning the forefoot-hindfoot spatial relationship. In producing more subtalar joint eversion, caused by the proximity of the midtarsal joint to the forces, the forefoot tended to evert at a higher degree than the subtalar joint. In order to align the plantar forefoot and hindfoot surfaces at the same plane, forces have to be applied to reinvert the forefoot relative to the rearfoot. This explained the rise in height of the medial toe areas and longitudinal arches, although the subtalar joint is at eversion. We found no significant difference in any of the other measured parameters when comparing the $4^{\circ}$ of eversion with the neutral position and $2^{\circ}$ of inversion.

Guidelines are usually followed to orientate the subtalar joint at a neutral position during molding for foot orthoses [7-8] because there is a common belief that the foot resting on the orthosis will be everted if the subtalar joint is aligned in such a way during foot impressions. The results of this study challenged this belief, showing that $4^{\circ}$ of eversion did not produce any significant changes in the shape of the plaster of Paris models as long as the plane of the first and fifth metatarsal heads was perpendicular to the bisector of the calcaneus.

The significant reduction of project volumes at $2^{\circ}$ of eversion suggested pronation of the hindfoot as well as the midfoot through the midtarsal joint. However, the differences in medial and longitudinal arch heights and navicular protrusions among all subtalar joint orientations were small. We found relatively larger changes in navicular heights. However, the changes were less than $1.5 \mathrm{~mm}$, which could be accommodated by a relief area on the orthosis. Changes in medial and lateral longitudinal arch heights were less than $0.6 \mathrm{~mm}$. Compared with the thickness of the plantar soft tissue at the metatarsal head, which 
has been reported to be over $5.5 \mathrm{~mm}$ [22], small changes in the heights of the arch support areas of an orthosis are likely to be compensated by the compressible plantar soft tissue. This led us to conclude that as long as the forefoot is appropriately aligned with respect to the hindfoot, different orientations of the subtalar joints would not have much effect on the configuration of the foot orthosis. A lack of understanding exists regarding how subtalar joint alignment during non-weight-bearing molding affects the shape of the molds obtained and its rationale, because the literature contains very little evidence. Therefore, this provides new information for clinicians that has not been published before.

Evaluating the geometry of positive foot models is important because it determines the shape of the orthosis. A positive model is usually evaluated by rulers measuring parameters such as foot length and heights of longitudinal arch $[16,19]$. These provide general information suggesting whether the orthosis will be of appropriate length and provide appropriate arch support. Digital scanning allows measurements of additional parameters in three dimensions. The projection volumes and mediolateral slopes along the long axis of the foot provide information on the three-dimensional spatial orientation of the different regions of the foot. The navicular height and protrusion suggests whether the relief to the bony prominence is at suitable position and adequate.

We found high reliability (ICC $=0.91$ ) measuring the subtalar joint angles during relaxed standing position. This corresponded well with a previous study [10] reporting intrarater and interrater reliabilities of 0.85 and 0.79 , respectively, when measuring the relaxed standing subtalar joint angles. A moderate interrater reliability (ICC = 0.60 ) of the subtalar joint angle measurement was reported when the subjects were in prone position. In the current study, the subtalar joint was aligned at different angles in prone lying position. The reliability of such alignment depended not only on the measurement method but also the amount of forces produced by the hands of the orthotist, which was not measured and might vary while awaiting the casting material to harden.

The main reason for choosing the non-weight-bearing molding method, which was commonly used in Australia and New Zealand [23], was to ensure that the forefoot could be aligned at $90^{\circ}$ to the rearfoot during the molding process at different subtalar joint orientations. The ability to assess forefoot correction and maintain the foot in its corrected position during foot molding is needed to provide an appropriate functional foot orthosis and cannot always be guaranteed using semi-weight-bearing molding techniques. Previous studies have found that foot molds with the same forefoot-hindfoot angle can be obtained using either the supine or prone non-weight-bearing approaches but not with the semi-weight-bearing approach [19]. The semi-weight-bearing approach is easier to perform, but the midtarsal joint cannot be adjusted using this method [19] because the foot is forced onto the floor. We found the reliability of capturing the forefoot-to-rearfoot relationship using the semi-weight-bearing approach to be lower than that using the non-weight-bearing method [2]. In addition, the foot orthosis produced from this method provides better medial longitudinal arch support [18]. This study therefore used foot molds under non-weight-bearing positions. Since foot molds taken in semi-weight-bearing positions could produce different forefoot-hindfoot angles [18], the results of this study could not be applied using semi-weight-bearing methods.

The results of this study also cannot be generalized to people with rigid foot deformities. While foot orthoses can be applied to rigid foot deformities such as those in rheumatoid feet and neuromuscular disorders, they are also frequently used to treat patients with flexible deformities such as flexible flat feet. Foot orthoses are also used to treat plantar fasciitis, which results from repetitive and excessive loads on the fascia and is not usually associated with a foot deformity. The outcomes of this study can only be applied to those without rigid foot deformities. This study investigated the effects of subtalar joint orientations on the shape of the positive model. It deserves further investigation to study the effects of various orientations of other segments of the lower limb.

\section{CONCLUSIONS}

Controversy exists as to how the subtalar joint should be aligned during foot molding for foot orthoses. This article evaluated the changes in foot shapes when the subtalar joint was aligned from $2^{\circ}$ of inversion to $4^{\circ}$ of eversion during non-weight-bearing foot molding. The results suggested that as long as the forefoot and hindfoot are aligned, variations in the orientation of the subtalar joint do not significantly change the plantar foot shape of the positive model. Because we performed foot molds only in non-weight-bearing positions, the results of this study 
could not be applied to the analysis of foot molds obtained in weight-bearing or semi-weight-bearing positions.

\section{ACKNOWLEDGMENTS}

\section{Author Contributions:}

Study concept and design: W. C. Lee, C. K. Lee, A. K. Leung.

Analysis and interpretation of data: W. C. Lee.

Acquisition of data: C. L. Lee.

Drafting of manuscript: W. C. Lee.

Critical revision of manuscript for important intellectual content:

A. K. Leung, S. W. Hutchins.

Statistical analysis: C. K. Lee.

Obtained funding: A. K. Leung.

Study supervision: A. K. Leung.

Financial Contributions: The authors have declared that no competing interests exist.

Funding/Support: This material was based on work supported by the Department of Health Technology and Informatics, The Hong Kong Polytechnic University.

Institutional Review: This study was approved by the Human Subjects Ethics Subcommittee of The Hong Kong Polytechnic University, and all subjects gave informed consent to this work.

Participant Follow-Up: The authors do not plan to inform participants of the publication of this study.

\section{REFERENCES}

1. Guldemond NA, Leffers P, Sanders AP, Emmen H, Schaper NC, Walenkamp GH. Casting methods and plantar pressure: Effects of custom-made foot orthoses on dynamic plantar pressure distribution. J Am Podiatr Med Assoc. 2006; 96(1):9-18. [PMID:16415278]

2. Laughton C, McClay Davis I, Williams DS. A comparison of four methods of obtaining a negative impression of the foot. J Am Podiatr Med Assoc. 2002;92(5):261-68. [PMID:12015406]

3. Ross AS, Jones LJ. Non-weightbearing negative cast evaluation. J Am Podiatry Assoc. 1982;72(12):634-38. [PMID:7175079]

4. Sobel E, Levitz SJ. Reappraisal of the negative impression cast and the subtalar joint neutral position. J Am Podiatr Med Assoc. 1997;87(1):32-33. [PMID:9009546]

5. McPoil TG, Knecht HG. Biomechanics of the foot in walking: A function approach. J Orthop Sports Phys Ther. 1985; 7(2):69-72. [PMID:18802288]

6. Leung AK, Cheng JC, Mak AF. Orthotic design and foot impression procedures to control foot alignment. Prosthet Orthot Int. 2004;28(3):254-62. [PMID:15658638]
7. Root ML, Weed JH, Orien WP. Neutral position casting techniques. Los Angeles (CA): Clinical Biomechanics Corporation; 1971.

8. Wright DG, Desai SM, Henderson WH. Action of the subtalar and ankle-joint complex during the stance phase of walking. J Bone Joint Surg Am. 1964;46:361-82. [PMID:14129684]

9. Van Gheluwe B, Kirby KA, Roosen P, Phillips RD. Reliability and accuracy of biomechanical measurements of the lower extremities. J Am Podiatr Med Assoc. 2002;92(6): 317-26. [PMID:12070231]

10. Sell KE, Verity TM, Worrell TW, Pease BJ, Wigglesworth J. Two measurement techniques for assessing subtalar joint position: A reliability study. J Orthop Sports Phys Ther. 1994; 19(3):162-67. [PMID:8156068]

11. Payne C, Chuter V, Oates M, Miller K. Introductory evaluation of a weightbearing neutral position casting device. J Podiatr Med. 2001;35:65-71.

12. Perry SD, Lafortune MA. Influences of inversion/eversion of the foot upon impact loading during locomotion. Clin Biomech (Bristol, Avon). 1995;10(5):253-57.

[PMID:11415562] http://dx.doi.org/10.1016/0268-0033(95)00006-7

13. McPoil TG, Cornwall MW. Relationship between three static angles of the rearfoot and the pattern of rearfoot motion during walking. J Orthop Sports Phys Ther. 1996; 23(6):370-75. [PMID:8727017]

14. Mannon K, Anderson T, Cheetham P, Cornwall MW, McPoil TG. A comparison of two motion analysis systems for the measurement of two-dimensional rearfoot motion during walking. Foot Ankle Int. 1997;18(7):427-31. [PMID:9252813]

15. Torburn L, Perry J, Gronley JK. Assessment of rearfoot motion: Passive positioning, one-legged standing, gait. Foot Ankle Int. 1998;19(10):688-93. [PMID:9801083]

16. Chuter V, Payne C, Miller K. Variability of neutral-position casting of the foot. J Am Podiatr Med Assoc. 2003; 93(1):1-5. [PMID:12533548]

17. Foulston J, Lord M, West S. Changes in plantar surface shape induced by corrective forefoot eversion. Clin Biomech (Bristol, Avon). 1990;5:229-35.

http://dx.doi.org/10.1016/0268-0033(90)90006-R

18. Tsung BY, Zhang M, Fan YB, Boone DA. Quantitative comparison of plantar foot shapes under different weightbearing conditions. J Rehabil Res Dev. 2003;40(6):517-26. [PMID:15077664] http://dx.doi.org/10.1682/JRRD.2003.11.0517

19. McPoil TG, Schuit D, Knecht HG. Comparison of three methods used to obtain a neutral plaster foot impression. Phys Ther. 1989;69(6):448-52. [PMID:2727068] 
20. Phillips RD, Christeck R, Phillips RL. Clinical measurement of the axis of the subtalar joint. J Am Podiatr Med Assoc. 1985;75(3):119-31. [PMID:3981442]

21. Woodburn J, Helliwell PS, Barker S. Three-dimensional kinematics at the ankle joint complex in rheumatoid arthritis patients with painful valgus deformity of the rearfoot. Rheumatology. 2002;41(12):1406-12. [PMID:12468821] http://dx.doi.org/10.1093/rheumatology/41.12.1406

22. Abouaesha FF, Van Schie CH, Griffths GD, Young RJ, Boulton AJ. Plantar tissue thickness is related to peak plantar pressure in the high-risk diabetic foot. Diabetes Care. 2001; 24(7):1270-74. [PMID:11423514] http://dx.doi.org/10.2337/diacare.24.7.1270

23. Landorf K, Keenan AM, Rushworth RL. Foot orthosis prescription habits of Australian and New Zealand podiatric physicians. J Am Podiatr Med Assoc. 2001;91(4):174-83. [PMID:11319247]
Submitted for publication March 18, 2011. Accepted in revised form September 26, 2011.

This article and any supplementary material should be cited as:

Lee WC, Lee CK, Leung AK, Hutchins SW. Is it important to position foot in subtalar joint neutral position during non-weight-bearing molding for foot orthoses? J Rehabil Res Dev. 2012;49(3):459-66.

http://dx.doi.org/10.1682/JRRD.2011.03.0049

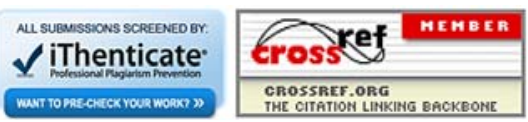

